PROCEEDINGS OF THE

AMERICAN MATHEMATICAL SOCIETY

Volume 135, Number 7, July 2007, Pages 2031-2035

S 0002-9939(07)08780-1

Article electronically published on February 28, 2007

\title{
UNIVERSALITY OF ASPLUND SPACES
}

\author{
PETR HÁJEK, GILLES LANCIEN, AND VICENTE MONTESINOS
}

(Communicated by Jonathan M. Borwein)

\begin{abstract}
Given any infinite cardinal $\tau$, there exists no Banach space of density $\tau$, which is Asplund or has the Point of Continuity Property and is universal for all reflexive spaces of density $\tau$.
\end{abstract}

In the framework of Banach space theory, the universal space problem can be traced back to the Scottish book [M], problem no. 49, which asks about the existence of a separable (resp. separable and reflexive or Asplund) Banach space containing all separable (resp. separable and reflexive or Asplund) spaces isomorphically. The case of separable spaces has a classical positive solution $C[0,1]$ (Banach and Mazur), while the reflexive or Asplund case has a negative solution (Szlenk $\underline{\underline{S}}$, resp. Wojtasczyk $[\mathbf{W}]$ ). In our present note we give a negative answer to the reflexive and Asplund situation for arbitrary densities, with a proof that also applies to the original problem. Our method of proof is essentially a blend of ideas from $[\mathrm{S}]$ and $[\mathrm{AB}$. It is perhaps less known that the Banach-Mazur theorem also generalizes for arbitrary densities. Yesenin-Volpin $[\mathrm{Y}]$ (assuming GCH) has shown the existence of a $C(K)$ space of density $\tau$, which contains all spaces of density $\tau$ isometrically, for every cardinal $\tau$. It is rather surprising that the WCG version of the question, which has been solved by Argyros and Benyamini (assuming GCH), has a positive answer if and only if $\operatorname{cof}(\tau)=\omega$. In particular, there exists a WCG space of density $\aleph_{\omega}$ containing isometrically and complementably all reflexive spaces of the same density, but there is no such reflexive space. There has been a large number of papers on the subject of universality (including various more specialized classes of spaces). Most notably, Bourgain [B] has shown that a Banach space universal for all separable reflexive spaces contains $C[0,1]$, and thus also every separable space. It follows from the construction in $\mathrm{AB}$ of a universal WCG space of density $\aleph_{\omega}$, that Bourgain's result does not generalize to arbitrary densities. We refer the reader to the upcoming monograph [HMVZ for a comprehensive list of results and references related to universal Banach spaces.

Our notation is standard, $\omega$ is the least infinite ordinal and $\tau$ stands for an arbitrary infinite cardinal, which we identify for convenience with the least ordinal of the same cardinality. Then, $\tau^{+}$denotes its successor cardinal. The main tool used in our proof will be a version of the Szlenk index (equivalent to the original index introduced by Szlenk in the case of spaces with a separable dual $[\mathrm{L}]$ ), which

Received by the editors January 17, 2006.

2000 Mathematics Subject Classification. Primary 46B30, 46 B03.

This work was supported by the following grants: Institutional Research Plan AV0Z10190503, A100190502, GA ČR 201/04/0090 and Project BMF2002-01423. 
is suitable also in the nonseparable setting (for a detailed account of results an applications of the Szlenk index see [L2]). The closed unit ball of a Banach space $X$ will be denoted $B_{X}$.

Definition 1. Let $X$ be an Asplund space, and let $B \subset X^{*}$ be a $w^{*}$-compact subset. Given $\varepsilon>0$, put $B_{0}^{\varepsilon}=B$. Proceeding inductively, if $\alpha$ is an ordinal, put

$$
B_{\alpha+1}^{\varepsilon}=B_{\alpha}^{\varepsilon} \backslash \bigcup W, \text { for all } W w^{*} \text {-open subsets of } B_{\alpha}^{\varepsilon} \text { with } \operatorname{diam}(W)<\varepsilon \text {. }
$$

If $\alpha$ is a limit ordinal, put

$$
B_{\alpha}^{\varepsilon}=\bigcap_{\beta<\alpha} B_{\beta}^{\varepsilon} .
$$

Clearly, the sets $B_{\alpha}^{\varepsilon}$ are $w^{*}$-compact. Assume that $\alpha$ is the least ordinal so that $B_{\alpha}^{\varepsilon}=\emptyset$. Then we define $S z^{\varepsilon}(B)=\alpha$. We define the Szlenk index $S z(B)=$ $\sup _{\varepsilon>0} S z^{\varepsilon}(B)$. In case when $B=B_{X^{*}}$, we abuse the notation slightly by denoting $S z^{\varepsilon}(X)=S z^{\varepsilon}(B)$ and calling $S z(X)=S z(B)$ the Szlenk index of the space $X$.

Few remarks are in order. It is clear that the Szlenk index of a Banach space $S z(X)$ is invariant under linear isomorphisms. The restriction of the definition to the class of Asplund spaces is not arbitrary. A well-known characterization of Asplund spaces (due to several authors, in particular Namioka, Phelps, Jayne and Rogers; see [DGZ, Thm. I.5.2]) claims that a Banach space is Asplund if and only if every $w^{*}$-compact subset of the dual has a nonempty $w^{*}$-open subset of diameter less than $\varepsilon$, for every $\varepsilon>0$. This is exactly the condition needed for the derivation process to end at some ordinal.

It is clear that $G \subset B \subset B_{X^{*}}$ implies that $S z(G) \leq S z(B)$. In particular, using the natural identifications, we immediately see that $S z(Z) \leq S z(X)$, whenever $Z$ is a linear quotient space of $X$. Moreover, we have the next lemma.

Lemma 2. Let $X$ be a Banach space, $Y \hookrightarrow X$. Then $S z(Y) \leq S z(X)$.

Proof. Denote $i: Y \rightarrow X$ as the embedding operator and let $\varepsilon>0$. Suppose that $P \subset B_{X^{*}}, S \subset B_{Y^{*}}$ are $w^{*}$-compact sets with $S \subset i^{*}(P)$. Then $S_{1}^{\varepsilon} \subset i^{*}\left(P_{1}^{\frac{\varepsilon}{2}}\right)$. Indeed, if $s \in S_{1}^{\varepsilon}$, then there exists a net $S \ni s_{\xi} \stackrel{w^{*}}{\rightarrow} s$, such that $\left\|s_{\xi}-s\right\| \geq \frac{\varepsilon}{2}$. Choose a net $p_{\xi} \in P, i^{*}\left(p_{\xi}\right)=s_{\xi}$. Since $P$ is $w^{*}$-compact, there exists a $w^{*}$ convergent subnet $p_{\zeta} \stackrel{w^{*}}{\rightarrow} p \in P$. Clearly, $i^{*}(p)=s$ and $\left\|p_{\zeta}-p\right\| \geq \frac{\varepsilon}{2}$ for all $\zeta$. So, we have that for every $w^{*}$-neighbourhood $W$ of $p$ in $P$, $\operatorname{diam}(W) \geq \frac{\varepsilon}{2}$. Thus $p \in P_{1}^{\frac{\varepsilon}{2}}$, and the claim follows. A standard induction argument now yields that $S z^{\varepsilon}(S) \leq S z^{\frac{\varepsilon}{2}}(P)$. We conclude the proof of the lemma by applying this result to $P=B_{X^{*}}$ and $S=B_{Y^{*}}$.

Lemma 3. Let $X$ be an Asplund space of density $\tau$. Then $S z(X)<\tau^{+}$.

Proof. To this end, it suffices to show that $S z^{\varepsilon}(X)<\tau^{+}$for every $\varepsilon>0$. Indeed (in ZFC, [J] p. 27]), $\tau^{+}>\omega$ is a regular cardinal, so $\operatorname{cof}\left(\tau^{+}\right)>\omega$, and $S z^{\varepsilon}(X) \stackrel{\varepsilon \rightarrow 0}{\longrightarrow}$ $S z(X)<\tau^{+}$. By Theorem I.5.2 of [DGZ], for every $w^{*}$-compact subset $K \subset X^{*}$, $K_{\alpha}^{\varepsilon}$ has a nonempty $w^{*}$-open subset disjoint with $K_{\alpha+1}^{\varepsilon}$. Thus $K \backslash K_{\alpha}^{\varepsilon}$ forms a strictly increasing long sequence of $w^{*}$-open subsets of $K$. Recall that $K$ has a basis of the $w^{*}$-topology of cardinality $\tau$, which implies that $K_{\alpha}^{\varepsilon}=\emptyset$ for some $\alpha<\tau^{+}$, as claimed. 
Recall that the height $\eta(K)$ of a topological space $K$ is the least ordinal $\beta$ for which the Cantor derivative $K^{(\beta)}$ is empty.

Lemma 4. Given an infinite cardinal $\tau$, for every $\alpha<\tau^{+}$there exists a strong Eberlein compact $K \subset c_{0}(\tau)$ with height at least $\alpha$.

Proof. Recall that a strong Eberlein compact is a subset $K \subset c_{0}(\tau)$, consisting of $\{0,1\}$-valued finitely supported functions, which is compact in the pointwise topology. It is easy to see that in such a compact, there does not exist any infinite sequence $A_{1} \varsubsetneqq A_{2} \varsubsetneqq \ldots$ of finite subsets of $\tau$, such that $\chi_{A_{i}} \in K$. Consequently, $K$ (hereditarily) has isolated points and is a scattered compact. Therefore the height $\eta(K)$ is well defined. Since $K$ may be viewed as a subset of $\tau^{<\omega}$, it is clear that $\eta(K)<\tau^{+}$. To construct $K$ with $\eta(K) \geq \alpha$, we proceed by induction. For $\alpha=1$, choose $K=\{0\} \cup\left\{\chi_{t}: t \in \tau\right\}$. Suppose we have constructed $K_{\beta} \subset c_{0}\left(\Gamma_{\beta}\right)$, $\eta\left(K_{\beta}\right) \geq \beta$ for all $\beta<\alpha<\tau^{+}$, where $\Gamma_{\beta}$ are pairwise disjoint index sets of cardinality $\tau$. Put $\Gamma=\bigcup \Gamma_{\beta}$, and $K=\left\{\chi_{A}:|A \backslash B| \leq 1\right.$, for some $B \subset A, \chi_{B} \in$ $\left.K_{\beta}, \beta<\alpha\right\}$. It is standard to verify that $K$ is a strong Eberlein compact. It follows that $|\Gamma| \leq|\tau \cdot \alpha|=\tau$ and $\eta(K) \geq \beta+1$ for all $\beta<\alpha$, and so $K$ satisfies $\eta(K) \geq \alpha$.

Theorem 5. Let $\tau$ be an infinite cardinal. Then for every $\tau \leq \alpha<\tau^{+}$there exists a reflexive Banach space $X_{\alpha}$ of density $\tau$ and such that $S z\left(X_{\alpha}\right) \geq \alpha$.

Proof. Let $K \subset c_{0}(\tau)$ be a strong Eberlein compact with $\eta(K) \geq \alpha$. By the DFJP] factorization theorem there exists a reflexive space $Y_{\alpha}$ (WLOG of density $\tau$ ) and a bounded linear and injective operator $T: Y_{\alpha} \rightarrow c_{0}(\tau), K \subset T\left(B_{Y_{\alpha}}\right)$. Being reflexive, $Y_{\alpha}$ is naturally a dual space, and so we have $S z\left(T^{-1}(K)\right) \leq S z\left(B_{Y_{\alpha}}\right)$. Besides, we have $\|f-g\|=1$ whenever $f, g \in K, f \neq g$. Thus there exists some $\varepsilon>0$ such that $\left\|T^{-1}(f)-T^{-1}(g)\right\| \geq 2 \varepsilon$ for all $f \neq g \in K$. It is now easy to verify that $T^{-1}\left(K^{(\beta)}\right) \subset\left(T^{-1}(K)\right)_{\beta}^{\varepsilon}$, and so we have $S z\left(X_{\alpha}\right)=S z\left(B_{Y_{\alpha}}\right) \geq$ $S z\left(T^{-1}(K)\right) \geq \alpha$, where $X_{\alpha}=Y_{\alpha}^{*}$.

Theorem 6. Given an infinite cardinality $\tau$, there exist no Asplund space of density $\tau$ universal for all reflexive (in particular also Asplund) spaces of density $\tau$.

Proof. By previous results, $S z(X)<\tau^{+}$for every Asplund space of density $\tau$. On the other hand, for every $\alpha<\tau^{+}$, there exists a reflexive space $X_{\alpha}$ of density $\tau$ and such that $S z\left(X_{\alpha}\right)>\alpha$. Lemma 2 finishes the proof.

We recall that a Banach space $X$ is said to have the Point of Continuity Property (in short PCP) if for every weakly closed and bounded subset $F$ of $X$ and every $\varepsilon>0$, the identity map from $(F, w)$ to $(F,\|\|)$ has a point of continuity. If $F \subset X$ is weakly closed, one can define a derived set $F_{[1]}^{\varepsilon}$ by deleting all the weakly open subsets of $F$ of diameter less than $\varepsilon$. Then, $S^{\varepsilon}(F)$ is the least ordinal $\alpha$ such that $S_{[\alpha]}^{\varepsilon}$ (defined inductively as before) is empty, $S(F)=\sup _{\varepsilon>0} S^{\varepsilon}(F)$ and (abusively) $S(X)=S\left(B_{X}\right)$.

Let $\tau$ be an infinite cardinal. If $X$ is a Banach space of density $\tau$ and with $\mathrm{PCP}$, then we clearly have that $S(X)<\tau^{+}$. Now, if $X$ is universal for all reflexive Banach spaces of density $\tau$, then, for any $\tau \leq \alpha<\tau^{+}, Y_{\alpha} \hookrightarrow X$, where $Y_{\alpha}$ is the reflexive space built in the proof of Theorem 5 . These observations clearly yield the following statement. 
Theorem 7. Given an infinite cardinality $\tau$, there exists no Banach space of density $\tau$ having PCP, that is universal for all reflexive (in particular, also RNP or $P C P)$ spaces of density $\tau$.

Final remarks. Our results can also be proved by extending Szlenk's construction after the first uncountable ordinal. This consists in defining $X_{\alpha}$ inductively as follows: $X_{0}=\ell_{2}, X_{\alpha+1}=X_{\alpha} \oplus_{1} \ell_{2}$ and $X_{\alpha}=\left(\sum_{\beta<\alpha} X_{\beta}\right)_{\ell_{2}}$ if $\alpha$ is a limit ordinal. The interest of our argument lies in the simple construction of an Eberlein compact space $K$ of height greater than $\alpha$, which is a concrete witness (through the factorization operator $T$ ) of the fact that $S z\left(X_{\alpha}\right) \geq \alpha$. We work directly with the dual balls of our spaces. This topological approach is in line with $[\mathrm{Y}], \mathrm{AB}]$ and most recently $\mathrm{Be}$. It brings up the following natural general question. Given a class of compact spaces, which is closed with respect to taking quotients, does there exist a universal element with respect to taking quotients of a given weight? Its "dual" version then reads: given a class of Banach spaces, closed with respect to taking subspaces, does there exists a universal space of a given density character? The "duality" here is in the sense that the dual unit ball of the Banach space, with its $w^{*}$-topology (which is always a compact space), belongs to the desired class of topological compact spaces. It seems that for classes of topological spaces which are fragmentable in some metric, there may be a chance to obtain a negative answer by building up an index theory along Szlenk's lines. If fragmentability is not present, another invariant has to be sought, or perhaps the answer is positive, as it is in the most general case of all compact spaces. We find particularly interesting the case of Corson compacta, not fragmentable in general ( $[\mathrm{AM},[\mathrm{F}]$ ), corresponding to the WLD Banach spaces ([FHHMPZ $]$ ).

\section{REFERENCES}

[AB] S. Argyros and Y. Benyamini, Universal WCG Banach spaces and universal Eberlein compacts, Isr. J. Math. 58 (1987), 305-320. MR0917361 (89d:46016)

[AM] S. Argyros and S. Mercourakis, On weakly Lindelöf Banach spaces, Rocky Mountain J. Math. 23 (1993), 395-446. MR.1226181 (94i:46016)

[Be] M. Bell, Universal uniform Eberlein compact spaces, Proc. AMS 128 (2000), 21912197. MR:1676311(2000m:54019)

[B] J. Bourgain, On separable Banach spaces universal for all separable reflexive spaces, Proc. AMS 79 (1980), 241-246. MR0565347 (81f:46021)

[DFJP] W. Davis, T. Figiel, W.B. Johnson and A. Pelczynski, Factoring weakly compact operators, J. Func. Anal. 17 (1974), 311-327. MR0355536 (50:8010)

[DGZ] R. Deville, G. Godefroy and V. Zizler, Smoothness and Renormings in Banach Spaces, Pitman Monographs 64 (1993). MR.1211634 (94d:46012)

[F] M. Fabian, Gateaux Differentiability of Convex Functions and Topology Canadian Math. Soc. Books, John Wiley (1997). MR1461271 (98h:46009)

[FHHMPZ] M. Fabian, P. Habala, P. Hájek, V. Montesinos, J. Pelant and V. Zizler, Functional analysis and infinite dimensional geometry, Canadian Math. Soc. Books, SpringerVerlag, (2001). MR.1831176 (2002f:46001)

[HMVZ] P. Hájek, V. Montesinos, J. Vanderwerff and V. Zizler, Biorthogonal systems in Banach spaces, monograph in preparation.

[J] T. Jech, Set theory, Academic Press (1978). MR0506523 (80a:03062)

[L] G. Lancien, Dentability indices and locally uniformly convex renormings, Rocky Mtn. J. Math. 23 (1993), 635-647. MR1226193 (94h:46026)

[L2] G. Lancien, A survey on the Szlenk index and some of its applications. To appear in RACSAM.

[M] R. D. Mauldin (Ed.), The Scottish Book, Birkhauser (1981). MR0666400|(84m:00015) 
[S] W. Szlenk, The non-existence of a separable reflexive Banach space, universal for all reflexive Banach spaces, Studia Math. 30 (1968), 53-61. MR0227743 (37:3327)

[W] P. Wojtasczyk, On separable Banach spaces containing all separable reflexive Banach spaces, Studia Math. 37 (1970), 197-202. MR0308750 (46:7864)

[Y] A. S. Yesenin-Volpin, On the existence of the universal bicompact of arbitrary weight, Dokl. Akad. Nauk 68 (1949), 649-652 (in Russian). MR0031534(11:165h)

Mathematical Institute, Czech Academy of Science, Žitná 25, 11567 Praha 1, Czech REPUBLIC

E-mail address: hajek@math.cas.cz

Université de Franche Comté, Besancon, 16, Route de Gray, 25030 Besancon Cedex, FRANCE

E-mail address: glancien@math.univ-fcomte.fr

Department of Applied Mathematics, Telecommunication Engineering Faculty, PolyTeChNic University of VAlencia, 46071 Valencia, Spain

E-mail address: vmontesi@mat.upv.es 